Proceedings of the WELCOME Scientific Meeting on Hybrid Nanostructures, Toruń, Poland, August 28-31, 2011

\title{
Electron Transmission through Graphene Bilayer Flakes
}

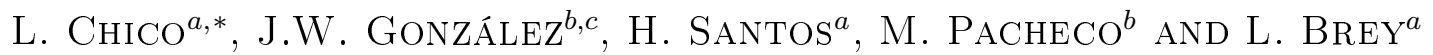 \\ ${ }^{a}$ Departamento de Teoría y Simulación de Materiales, Instituto de Ciencia de Materiales de Madrid \\ Consejo Superior de Investigaciones Científicas, Cantoblanco, 28049 Madrid, Spain \\ ${ }^{b}$ Departamento de Física, Universidad Técnica Federico Santa María, Casilla 110 V, Valparaíso, Chile \\ ${ }^{c}$ International Iberian Nanotechnology Laboratory, Av. Mestre José Veiga, 4715-310 Braga, Portugal
}

We investigate the electronic transport properties of a bilayer graphene flake contacted by two monolayer nanoribbons. This finite-size bilayer flake can be built by overlapping two semi-infinite ribbons. We study and analyze the electronic behavior of this structure by means of a tight-binding method and a continuum Dirac model. We have found that the conductance oscillates markedly between zero and the maximum value of the conductance, allowing for the design of electromechanical switches.

PACS: 73.22.-f, 73.63.-b

\section{Introduction}

Graphene is a one-atom thick electronic material; it is a stable pure two-dimensional system, successfully isolated in $2005[1,2]$, composed by a covalent-bond carbon monolayer sheet packed in a honeycomb crystal lattice with two inequivalent triangular sublattices $A$ and $B$.

Carriers in monolayer graphene behave as two-dimensional (2D) massless Dirac fermions [3], with a linear dispersion relation $\varepsilon(\boldsymbol{k})= \pm v_{\mathrm{F}} k$. Phenomena of fundamental nature, such as quantum Hall effect $[1,2]$ and Klein tunneling [4] have been recently measured in graphene based devices. Graphene is also regarded as a promising candidate for nanoelectronic applications. By patterning graphene, the electronic structure can be altered $[5,6]$. Another way to modify the band structure of graphene is to stack two graphene monolayers, forming bilayer graphene $[7,8]$. Graphene bilayer systems show interesting properties with strong dependence on stacking. In bilayer graphene there are four atoms per unit cell, with inequivalent sites $A 1, B 1$ and $A 2, B 2$ in the first and second graphene layers, respectively.

In this work we consider narrow graphene nanoribbons in the energy range for which only one incident electronic channel is active. We calculate the conductance with two different approaches: a tight-binding model using the Landauer-Büttiker formalism and a mode-matching calculation in the continuum Dirac-like Hamiltonian approximation. By imposing appropriate boundary conditions, the physics of graphene nanoribbons is well described within a continuum Dirac model $[9,10]$.

\section{Theoretical description of the system}

The low energy properties in graphene are mainly determined by the carbon $p_{z}$ orbitals. We adopt a

\footnotetext{
* corresponding author; e-mail: leonor.chico@icmm.csic.es
}

$\pi$-band tight-binding Hamiltonian with nearest-neighbor in-plane interaction given by the hopping parameter $\gamma_{0}=$ $2.66 \mathrm{eV}$. In undoped graphene, the conduction and valence bands touch at two inequivalent points of the Brillouin zone $\boldsymbol{K}$ and $\boldsymbol{K}^{\prime}$. Near these points, the electric properties of graphene can be described by a massless Dirac Hamiltonian [3]. As to bilayer graphene, it consists of two graphene layers coupled by tunneling. The interlayer coupling is modeled with a single hopping $\gamma_{1}$ connecting atoms directly on top of each other, which we take as $\gamma_{1}=0.1 \gamma_{0}$, in agreement with experimental results [11].

\subsection{Tight-binding Hamiltonians}

The Hamiltonians used to describe the behavior of both considered stacks are similar to those used in previous works [12, 13]. Different stacking orders can occur in bilayer graphene. The most commonly studied is $A B$ stacking. Here, the layers are arranged in such a way that the $A 1$ sublattice is exactly on top of the sublattice $B 2$. In the $A A$ stacking, both sublattices of one sheet $A 1$ and $B 1$, are located directly on top of the two sublattices of the other sheet $A 2$ and $B 2$.

As we are interested in the transport properties of bilayer flakes, we will concentrate on structures where the leads are monolayer armchair graphene nanoribbons (aGNR), with widths chosen to have metallic character. We choose a configuration where the left lead is connected to the bottom layer of the flake, whereas the right lead is connected to the top layer of the flake, as depicted in Fig. 1a. The atomic geometry of the monolayer aGNR leads and the corresponding low energy electronic bands are shown in Fig. 1b.

The low energy spectra of bilayer nanoribbons depend on the particular stacking. In Fig. 1c we plot the tight-binding band structure of a bilayer nanoribbon with $A A$ stacking. The bands also present a linear dispersion and they can be understood as bonding/antibonding combinations of the constituent monolayer aGNR bands. The 
(a)

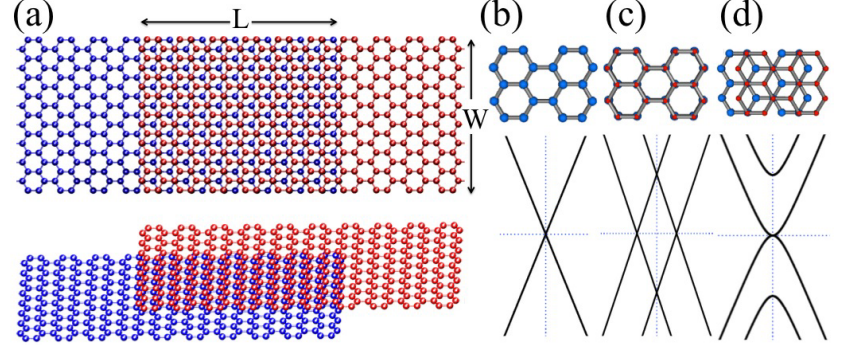

Fig. 1. (a) The bilayer graphene flake is formed by the overlap of two semi-infinite nanoribbons. The width and length of the bilayer region are $W$ and $L$, respectively. (b)-(d) Atomic structure geometries and band dispersion relations around the Dirac point for several armchair-terminated nanoribbons. The ribbon longitudinal axes are in the horizontal direction. (b) Monolayer armchair; (c) bilayer nanoribbon with $A A$ stacking; (d) bilayer ribbon with $A B$ stacking. For this energy range, the dispersion relations (b)-(d) are independent of the ribbon width.

$A B$ stacking can be achieved from the $A A$ bilayer geometry by displacing one graphene monolayer with respect to the other, in such a way that the atoms of one sublattice (i.e., $A$ ) of the top monolayer are placed over the atoms of the other sublattice $(B)$ of the bottom monolayer. This is schematically shown in the upper part of Fig. 1d. The band structure of metallic $A B$-stacked bilayer graphene nanoribbons have a parabolic dispersion at the Dirac point, as we can see in Fig. 1d.

Due to the lack of translational invariance of the system, in the tight binding model we calculate the electronic and transport properties using the surface Green function matching method [14, 15]. To this end, the system is partitioned in three blocks: two semi-infinite leads, which we assume to be semi-infinite aGNR, and the conductor, consisting of the bilayer flake. The Hamiltonian is $H=H_{\mathrm{C}}+H_{\mathrm{R}}+H_{\mathrm{L}}+V_{\mathrm{LC}}+V_{\mathrm{RC}}$, where $H_{\mathrm{C}}, H_{\mathrm{L}}$, and $H_{\mathrm{R}}$ are the Hamiltonians of the central portion, left and right leads respectively, and $V_{\mathrm{LC}}, V_{\mathrm{RC}}$ are the coupling matrix elements from the left $\mathrm{L}$ and right $\mathrm{R}$ lead to the central region $\mathrm{C}$. The Green function of the conductor is $\mathcal{G}_{\mathrm{C}}(E)=\left(E-H_{\mathrm{C}}-\Sigma_{\mathrm{L}}-\Sigma_{\mathrm{R}}\right)^{-1}$, where $\Sigma_{\ell}=V_{\ell \mathrm{C}} g_{\ell} V_{\ell \mathrm{C}}^{\dagger}$ is the selfenergy due to lead $\ell=\mathrm{L}, \mathrm{R}$, corresponding to the left or right side of the conductor and $g_{\ell}=\left(E-H_{\ell}\right)^{-1}$ is the Green function of the semi-infinite lead $\ell$.

In the linear response regime, the conductance can be calculated within the Landauer formalism as a function of the energy $E$. In terms of the Green function of the system $[14,16]$, it reads

$$
G=\frac{2 e^{2}}{h} T(E)=\frac{2 e^{2}}{h} \operatorname{Tr}\left(\Gamma_{\mathrm{L}} G_{\mathrm{C}} \Gamma_{\mathrm{R}} G_{\mathrm{C}}^{\dagger}\right),
$$

where $T(E)$ is the transmission function across the conductor, and $\Gamma_{\ell}=\mathrm{i}\left[\Sigma_{\ell}-\Sigma_{\ell}^{\dagger}\right]$ is the coupling between the conductor and the $\ell=\mathrm{L}, \mathrm{R}$ lead.

\subsection{Dirac-like Hamiltonians}

Most of the low energy properties of monolayer and bilayer graphene nanoribbons can be understood using a $\boldsymbol{k} \cdot \boldsymbol{p}$ approximation, which yields a Dirac-like Hamiltonian [9]. We have found analytical expressions for both stacks ( $A A$ and $A B$ ), but we only give here those for the $A A$-stacking, because they are much simpler and we can analyze through them the energy dependence, interlayer coupling, and superposition length [12].

The low-energy effective bilayer Hamiltonian describing the properties of an infinite $A A$-stacked bilayer has the form

$$
H_{A A}=\left(\begin{array}{cccc}
0 & v_{\mathrm{F}} \pi^{\dagger} & \gamma_{1} & 0 \\
v_{\mathrm{F}} \pi & 0 & 0 & \gamma_{1} \\
\gamma_{1} & 0 & 0 & v_{\mathrm{F}} \pi^{\dagger} \\
0 & \gamma_{1} & v_{\mathrm{F}} \pi & 0
\end{array}\right),
$$

where $\pi=k_{x}+\mathrm{i} k_{y}=k \mathrm{e}^{\mathrm{i} \theta_{k}}, \theta_{\boldsymbol{k}}=\tan ^{-1}\left(k_{x} / k_{y}\right)$, $v_{\mathrm{F}}=\frac{\sqrt{3}}{2} \gamma_{0} a_{0}$, where $a_{0}=2.46 \AA$ is the graphene in-plane lattice parameter, and $\boldsymbol{k}=\left(k_{x}, k_{y}\right)$ is the momentum relative to the Dirac point. The Hamiltonian acts on a four-component spinor $\left[\phi_{A}^{(1)}, \phi_{B}^{(1)}, \phi_{A}^{(2)}, \phi_{B}^{(2)}\right]$. The eigenfunctions of this Hamiltonian are bonding and antibonding combinations of the isolated graphene sheet solutions,

$$
\varepsilon_{s, \pm}^{A A}=s v_{\mathrm{F}} k \pm \gamma_{1}, \quad \psi_{s, \pm}^{A A}=\left(\begin{array}{c}
1 \\
s \mathrm{e}^{\mathrm{i} \theta_{\boldsymbol{k}}} \\
\pm 1 \\
\pm s \mathrm{e}^{\mathrm{i} \theta_{\boldsymbol{k}}}
\end{array}\right) \mathrm{e}^{\mathrm{i} \boldsymbol{k} \cdot \boldsymbol{r}}
$$

with $s= \pm 1$.

In accordance with the geometry shown in Fig. 1, we assume for nanoribbons that the system is invariant in the $x$ direction, and therefore $k_{x}$ is a good quantum number. In the case of metallic aGNR, the boundary conditions are satisfied [9] for $k_{y}=0$ independently of the nanoribbon width; this $k_{y}=0$ state is the lowest energy band confined in the aGNR. We have checked that the dispersion of the lowest energy band obtained by solving the Dirac model coincides with that obtained by diagonalizing the tight-binding Hamiltonian for the monolayer and $A A$-stacked bilayer nanoribbons. Therefore, the Dirac approximation is a good description for the low energy properties of these nanoribbons, Fig. 1b-d.

In the low-energy limit, we can obtain the conductance of the system by matching the eigenfunctions of the Dirac-like Hamiltonians. We consider incident electrons from the lowest energy subband, which corresponds to a transversal momentum $k_{y}=0$ in aGNRs. Assuming an electron with energy $E$ coming from the left monolayer ribbon, we compute the transmission coefficient $t$ to the right monolayer lead. In the central part the wavefunctions are linear combinations of the solutions of the bilayer nanoribbon Hamiltonians. The transmission, reflection and the coefficients of the wavefunctions in the bilayer part are determined by imposing the appropriate boundary conditions at the beginning $(x=0)$ and at the end $(x=L)$ of the bilayer region. Matching of the 
wave functions amounts to require their continuity. As the Hamiltonian is a first-order differential equation, current conservation is ensured automatically. The precise boundary condition depends on the lead configuration and on the stacking.

\section{Results}

In the $A A$ stacking, the dispersion in the central part is given by Eq. (3), and for each incident carrier with momentum $k_{x}$, there are always two reflected and two transmitted eigenfunctions with momenta $\pm\left(k_{x} \pm \gamma_{1} / v_{\mathrm{F}}\right)$; see Fig. 1c.

In particular, for the chosen leads configuration, the bottom wave function $\phi_{A}^{(1)}(x)$ and the top wave function $\phi_{B}^{(2)}(x)$ should be continuous at $x=0$ and $x=L$, respectively. In addition, the hard-wall condition should be satisfied, $\phi_{A}^{(2)}(x=0)=\phi_{B}^{(1)}(x=L)=0$. These boundary conditions yield the transmission

$$
T=1-\frac{\cos ^{4} \frac{\gamma_{1} L}{v_{\mathrm{F}}}}{2\left(1-\cos \frac{2 E L}{v_{\mathrm{F}}}\right) \sin ^{2} \frac{\gamma_{1} L}{v_{\mathrm{F}}}+\cos ^{4} \frac{\gamma_{1} L}{v_{\mathrm{F}}}} .
$$

We see from these equations that the conductance changes periodically as function of the incident energy and length of the bilayer flake. For fixed $L$, the transmission is a periodic function of the incident energy. In this geometry there are antiresonances, $T=0$, at energies given by $\frac{\pi v_{\mathrm{F}}}{L} n$, with $n=0,1,2 \ldots$ These energies correspond to quasilocalized states in the top part of the bilayer flake. The paths through the bottom graphene ribbon and through the quasilocalized state of the top flake interfere destructively, producing the antiresonance [17-19].

For fixed energy, the conductance varies periodically with the length of the bilayer flake. There is a period, $\frac{\pi v_{\mathrm{F}}}{E}$, related to the energy of the incident carrier; other periods are harmonics of that imposed by the interlayer hopping, $\frac{\pi v_{\mathrm{F}}}{\gamma_{1}}$. The dependence of the conductivity on $\gamma_{1}$ can be understood by resorting to a simple non-chiral model with linear dispersion. Consider an incident carrier from the left with momentum $k_{x}$ and energy $E=v_{\mathrm{F}} k_{x}$ in the bottom sheet. When arriving at the bilayer central region, the incident wavefunction decomposes into a combination of bonding $(b)$ and antibonding $(a)$ states of the bilayer with momentum $k^{b(a)}=k_{x} \pm \frac{\gamma_{1}}{v_{\mathrm{F}}}$. The conductance through the bilayer region is proportional to the probability of finding an electron at the top (bottom) end of the central region, $1+\cos \left(k^{b}-k^{a}\right) L=1+\cos \frac{\gamma_{1} L}{v_{\mathrm{F}}}$. This simple model explains the dependence of the conductivity on harmonics of $\cos \frac{\gamma_{1} L}{v_{\mathrm{F}}}$.

Let us recall here that the length of a unit cell (u.c.) for an armchair ribbon is $3 a_{\mathrm{CC}}=\sqrt{3} a_{0}$. In the following figures, we choose to give the system length $L$ in terms of the armchair ribbon u.c. length, which is unambiguous for the discrete tight-binding model. Note that, in the continuum approximation, the hard wall conditions at the edges of the system $(x=0$ and $x=L)$ are set at two extra rows of atoms where the wave functions are imposed to be zero. This amounts to add to the system length the quantity $a_{\mathrm{CC}}$, which we take into account when comparing the continuum and the tight-binding results.

As discussed in the previous section, the expression for the transmission Eq. (4) demonstrates that the dependence with the system length has periodicities related to the interlayer coupling $\gamma_{1}$. This is evident in Figs. 2 and 3 , which shows the length and incident energy dependence of the conductance for the stackings $A A$ and $A B$. The tight-binding calculations are performed for a ribbon of width $N=11$, but for this energy range only one channel contributes to the conductance in the monolayer and at most two channels in the bilayer flake, so the conductance is independent of $N$.
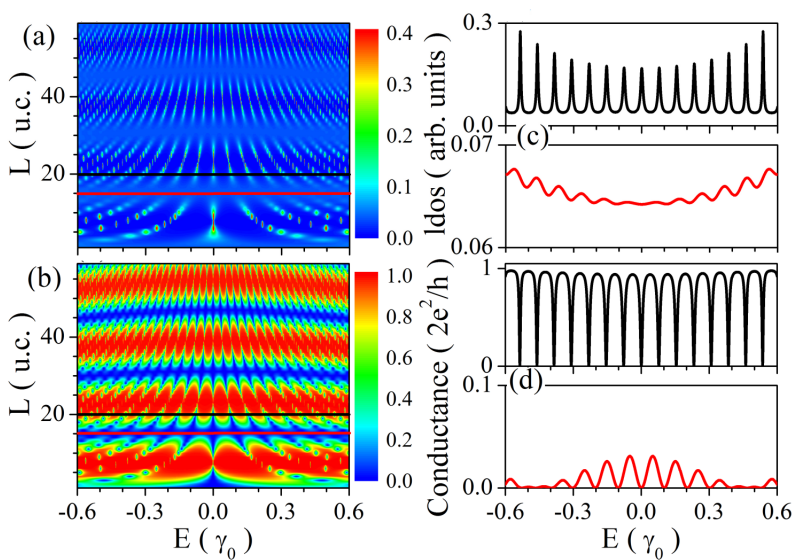

Fig. 2. Conductance and local density of states for $A A$-stacked bilayer as a function of the Fermi energy $E$ and bilayer region length $L$ for a ribbon of width $N=11$. (a, c) Local density of states; (b, d) conductance. In both contour plots, transverse cuts have been made, (c, d), which correspond to the selection of specific lengths in the contour plots to examine in detail, in this case, black is for $L=15$ and red is for $L=20$. For purposes of clarity, we have modified the scales in parts $(c, d)$.

As expected, the $A A$ stacking shows clear antiresonances in the conductance as a function of length and energy, Fig. 2b-d, since in the whole range of energies, there are always two available channels, as shown in Fig. 1c. These conductance antiresonances correspond to peaks in the local density of states (LDOS), Fig. $2 \mathrm{a}-\mathrm{c}$.

The conductance shows one clear length period of 15 u.c., stemming from the $\cos \frac{2 E L}{v_{\mathrm{F}}}$ term in the conductance, which appears in the analytical expression Eq. (4).

On the other hand, the $A B$-stacked bilayer graphene presents two energy regions with different behaviors (Fig. 3). At low energy range $E<\gamma_{1}$ there is only one transmission channel in the bilayer. Thus, although the conductance oscillates due to finite-size effects, there are no conductance antiresonances for the $A B$ stacking at this energy. However, for $E \geq \gamma_{1}$, the $A B$ case now 

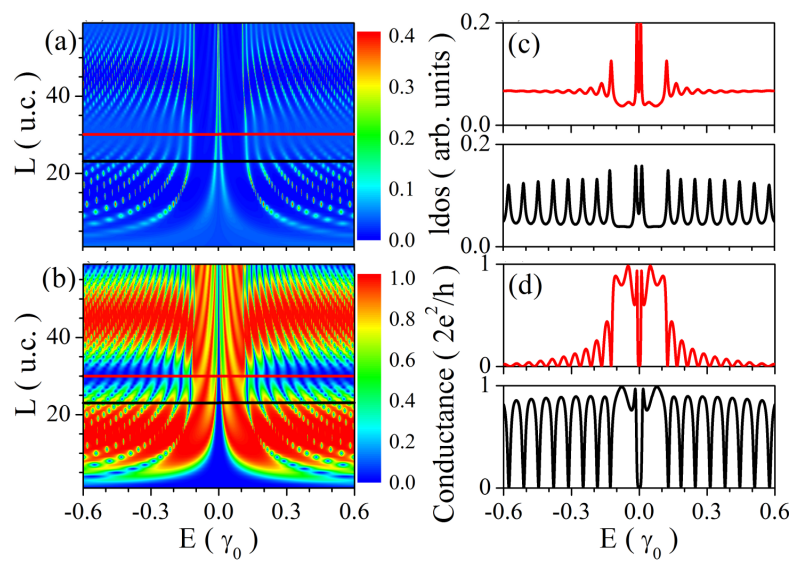

Fig. 3. Conductance and local density of states for $A B$-stacked bilayer as a function of the Fermi energy $E$ and bilayer region length $L$ for a ribbon of width $N=11$. (a, c) Local density of states; (b, d) conductance. In both contour plots (a) and (b), two transversal cuts have been made, (c, d), corresponding to two different lengths, namely, $L=23$ (in red) and $L=30$ (in black). For the sake of clarity, we have modified the scales in part (c).

presents antiresonances with zero conductance, as for the $A A$ stacking. This is due to the presence of an additional conductance channel at this energy range.

The conductance in the $A B$ case shows one clear length period close to 30 u.c. This period doubles that of the $A A$ stacking; and it can be related to the fact that in the $A B$ stacking only half of the atoms have interlayer coupling, thus having an effective coupling smaller by a factor of two.

As discussed before, the most characteristic feature of the transmission is the appearance of the Fano antiresonances with zero conductance. This can happen for any energy in the case of $A A$ stacking because there are always two conducting channels in the bilayer. On the contrary, for $A B$ stacking, with only one channel for $E<\gamma_{1}$, the oscillations in the conductance are due to a FabryPérot-like effect in this energy range, i.e., the interference of one scattering channel with itself due to the finite size of the structure. Therefore, the $A B$ stacking presents non-zero minima in the conductance in the $\left(0, \gamma_{1}\right)$ energy range, whereas the antiresonances above $\gamma_{1}$ clearly reach zero values.

We clearly see the main transmission antiresonances with a 15 u.c. length period in $A A$ and 30 u.c. length period for the $A B$. In fact, it turns out that for certain flake sizes $L$, the conductance is zero, independently of the energy. As this spatial period depends directly on the interlayer coupling strength $\gamma_{1}$, we propose that this feature can be used to estimate the interlayer hopping parameter, the value of which is still under debate [20], by overlapping two nanoribbons and displacing one of them with respect to the other, the spatial period could be measured and thus $\gamma_{1}$ would be obtained.
As the variation of the conductance as a function of length is so dramatic, from one quantum of conductance to zero, this system can function as an electromechanical switch, turning from the maximum conductance to zero by a displacement of a few $\AA$.

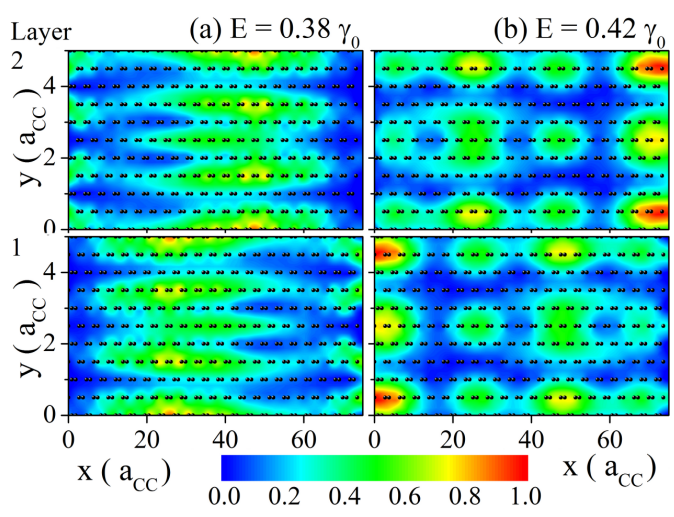

Fig. 4. Local density of states for a bilayer in the $A A$ stacking, for a nanoribbon $N=11$ and a bilayer length $L=20$, for energies corresponding to a peak in the density of states $\left(E=0.38 \gamma_{0}\right)$, and a minimum in the LDOS $\left(E=0.42 \gamma_{0}\right)$. In this plot, the left lead is located on the left edge of layer 1 , and the right lead is located on the right side of layer 2 .

In order to understand the electronic behavior of the system, we analyze the spatial distribution of the local density of states, selecting two energies with well-defined behaviors, given the clear resonances that presents the system with $A A$ stacking for $L=20$ (Fig. 2c). We choose a peak in the density of states, at $E=0.38 \gamma_{0}$ (Fig. 4a) and a minimum in the LDOS at $E=0.42 \gamma_{0}$ (Fig. $4 \mathrm{~b}$ ). These energies correspond to a drop in the conductance in the first case, and the second corresponds to an energy where the conductance is close to the maximum value. Notice that in both cases there is a symmetry in the LDOS distribution under the interchange of the two layers. For the first energy, $E=0.38 \gamma_{0}$ (Fig. 4a) corresponding to a peak in the density of states and a drop in conductance, this behavior is caused by an antiresonance in the flake. There is a quasi-confined state in the flake, but it is not connected to the leads, because the LDOS has two minima in the connection to the leads, thus producing a drop in the conductance. However, for $E=0.42 \gamma_{0}$ (Fig. 4b), the situation is just the opposite: the density of states has an overall local minimum, but the leads are well connected, leading to a transmission maximum.

\section{Summary}

In this work, we have studied the conductance of a graphene bilayer flake contacted by two monolayer nanoribbons. Two different stackings for the graphene flake have been taken into account, namely, $A A$ and $A B$.

We have calculated the conductance with a tight-binding approach and also by performing a mode- 
-matching calculation within the continuum Dirac model, by choosing the appropriate boundary conditions. We have explained the features in the transmission and obtained analytical expressions that allow us to elucidate the transport characteristics of these structures. We have found several periodicities on the conductance and in the local density of states, related to the energy and the interlayer coupling of the system.

In particular, for the $A A$ configuration, we have found that the conductance through the flake shows the Fano-like antiresonances, that we have related to the interference of two different propagating channels in the structure. For a flake of length $L$, the main transmission period is given by $\pi v_{\mathrm{F}} / L$. For a fixed incident energy, the conductance as a function of the system length $L$ oscillates with two main periods related to the energy $E$ and the interlayer coupling $\gamma_{1}$.

For the $A B$ stacking, we have found two distinct behaviors as a function of the incident energy $E$ : for energies larger than the interlayer hopping $\gamma_{1}$, the transmissions resemble those found for the $A A$ stacking. This is due to the existence of two propagating channels at this energy range. There is, however, a difference on the main period related to the interlayer hopping $\gamma_{1}$, which is twice the period found for the $A A$ stacking. This can be understood by noticing that in the $A B$ stacking only half of the atoms are connected between the two graphene layers. For energies smaller than $\gamma_{1}$, the $A B$-stacked flake only has one eigenchannel, and the conductance shows resonances related to the existence of the Fabry-Pérot-like states in the system.

The conductance of these bilayer flakes can oscillate between zero and the maximum conductance as a function of length; thus, a system composed by two overlapping nanoribbons can behave as an electromechanical switch. We propose that these characteristics can be employed to measure the interlayer hopping in bilayer graphene. Our results give a comprehensive view of transport through bilayer graphene flakes, clarifying the role of stacking, contact geometries, flake width and length in the conductance of these structures.

\section{Acknowledgments}

This work has been partially supported by the Spanish DGES grant FIS2009-08744 and CSIC-CONICYT grant 2009CL0054. J.W.G. and M.P. would like to gratefully acknowledge helpful discussion to Dr. L. Rosales. M.P. acknowledges the financial support of FONDECYT 1100672 and DGIP/USM 11.11.62 internal grant.

\section{References}

[1] K.S. Novoselov, D. Jiang, T. Booth, V.V. Khotkevich, S.M. Morozov, A.K. Geim, Nature 438, 197 (2005).

[2] Y. Zhang, Y.-W. Tan, H.L. Stormer, P. Kim, Nature 438, 201 (2005).

[3] A.H. Castro-Neto, F. Guinea, N.M.R. Peres, K.S. Novoselov, A.K. Geim, Rev. Mod. Phys. 81, 109 (2009).

[4] A.F. Young, P. Kim, Nature Phys. 5, 222 (2009).

[5] K. Nakada, M. Fujita, G. Dresselhaus, M.S. Dresselhaus, Phys. Rev. B 54, 17954 (1996).

[6] M. Fujita, K. Wakabayashi, K. Nakada, K. Kusakabe, J. Phys. Soc. Jpn. 65, 1920 (1996).

[7] E. McCann, V.I. Fal'ko, Phys. Rev. Lett. 96, 086805 (2006).

[8] E.V. Castro, K.S. Novoselov, S.V. Morozov, N.M.R. Peres, J.M.B. Lopes dos Santos, J. Nilsson, F. Guinea, A.K. Geim, A.H. Castro Neto, Phys. Rev. Lett. 99, 216802 (2007).

[9] L. Brey, H.A. Fertig, Phys. Rev. B 73, 235411 (2006).

[10] A.R. Akhmerov, C.W.J. Beenakker, Phys. Rev. B 77, 085423 (2008).

[11] T. Ohta, A. Bostwick, T. Seyller, K. Horn, E. Rotenberg, Science 313, 951 (2006).

[12] J.W. González, H. Santos, M. Pacheco, L. Chico, L. Brey, Phys. Rev. B 81, 195406 (2010).

[13] J.W. González, H. Santos, E. Prada, L. Brey, L. Chico, Phys. Rev. B 83, 205402 (2011).

[14] L. Chico, L.X. Benedict, S.G. Louie, M.L. Cohen, Phys. Rev. B 54, 2600 (1996).

[15] J.W. González, L. Rosales, M. Pacheco, Physica B, Condens. Matter 404, 2773 (2009).

[16] S. Datta, Electronic Transport in Mesoscopic Systems, Cambridge University Press, Cambridge 1995.

[17] E. Tekman, P.F. Bagwell, Phys. Rev. B 48, 2553 (1993).

[18] P.A. Orellana, F. Domínguez-Adame, I. Gómez, M.L. Ladrón de Guevara, Phys. Rev. B 67, 085321 (2003).

[19] J.W. González, M. Pacheco, L. Rosales, P.A. Orellana, Phys. Rev. B 83, 155450 (2011).

[20] J. Nilsson, A.H. Castro Neto, N.M.R. Peres, F. Guinea, Phys. Rev. B 73, 214418 (2006). 